\title{
Community Workforce Differences in Mental Health Across Gender and Job Description in Southwest Indiana
}

\author{
Victoria Rone ${ }^{1}$, Majed Koleilat ${ }^{2}$, Kara Garcia ${ }^{3}$ \\ ${ }^{1}$ Indiana University School of Medicine; ${ }^{2}$ Research Institute of Deaconess Clinic; ${ }^{3}$ Indiana University \\ School of Medicine, Department of Radiology \& Imaging Sciences
}

Background and Hypothesis: In May 2020, a community partnership was built in Southwest Indiana to determine the local prevalence of COVID-19 to guide reopening in the region. One year later, a follow-up survey study was launched to address new, pressing questions related to mental health in the community workforce. We hypothesized that adverse mental health symptoms would be disproportionately experienced by specific worker groups based on degree of in-person contact over the past year and demographic characteristics such as age and gender.

Methods: For the 2021 survey, participants were invited through their place of employment or via phone to participate in a short online survey consisting of questions related to demographics, general work roles, company policies over the past year, and wellness. When examining mental health, the Patient Health Questionnaire-2 (PHQ-2) and General Anxiety Disorder-2 (GAD-2) were used to determine feelings of depression and anxiety, respectively.

Results: 480 participants filled out the 2021 online survey (73\% of which were returning). 442 of these responses were complete and eligible for analysis of mental health and degree of inperson contact. 345 responses were complete and eligible for information regarding age. No correlation was found between age and mental health. However, differences were observed based on gender and job description. Women $(n=244)$ and customer-facing employees $(n=99)$ reported more frequent symptoms of anxiety and depression, with customer-facing women reporting the most. Comparing only employees that did not work remotely during the pandemic, customer-facing employees still reported more frequent symptoms of anxiety and depression.

Conclusion and Potential Impact: The impacts of COVID-19 on the workforce are still being studied, but the pandemic continues to take its toll. These mental health trends will help guide future policies to support and improve employee wellbeing, both within participating companies and the workforce at large. 